\title{
Cernambi Virgem Ecológico (CVE), un nuevo caucho de extracción natural con calidad y directo de la selva amazónica a la industria del calzado
}

\author{
Francisco Samonek'; Miguel Y. Ramírez-Sánchez ${ }^{2,3^{\star}}$, y Régis A. Paiva ${ }^{4}$. \\ (1) UNINI, Universidad Internacional Iberoamericana, Brasil (correo-e: franciscosamonek@yahoo.com.br) \\ (2) UNIC, Universidade Internacional do Cuanza, Angola \\ (3) UNINI, Universidad Internacional Iberoamericana, México (correo-e: miguelyramirez@hotmail.com) \\ (4) Polo de Proteção da Biodiversidade e Uso Sustentável dos Recursos Naturais (POLOPROBIO): Alameda Fabio \\ Miranda, 937, Castanhal, Pará-Brasil (correo-e: regispaivaac@gmail.com).
}

* Autor a quien debe ser dirigida la correspondencia

Recibido Mar. 30, 2021; Aceptado Jun. 3, 2021; Versión final Ago. 3, 2021, Publicado Dic. 2021

\begin{abstract}
Resumen
El principal objetivo de esta investigación es analizar las propiedades del caucho Cernambi Virgem Ecológico (CVE) recogido por productores en Brasil. El caucho cultivado ha puesto fin a la extracción en la selva Amazónica por falta de competitividad. EI CVE es un nuevo caucho, de pequeños coágulos, limpios e inodoros, hechos de látex cosechado diariamente. Un caucho más fácil de fabricar, de buena calidad, con valor añadido y que va directamente del cauchero a la industria. Según las pruebas realizadas, los resultados muestran que la curva representativa de tensión-deformación obtenida para el CVE tiene un límite de resistencia a la tracción de $0.54 \mathrm{MPa}$ y una deformación específica con cerca de 767\%. El CVE tiene una calidad equivalente al Granulado Escuro Brasileiro (GEB) y a la Ribbed Smoked Sheet (RSS). Se concluye que el CVE puede eliminar perfectamente la fase de pre-preparación de la goma cruda fabricada en las plantas GEB.
\end{abstract}

Palabras clave: caucho extractivo; desarrollo sustentable; medio ambiente; industria; calzado; servicio ambiental

\section{Cernambi Virgem Ecológico (CVE), a new quality natural extraction rubber, directly from the Amazon rainforest to the footwear industry}

\begin{abstract}
The primary objective of this research study is to characterize the properties of the Cernambi Virgem Ecológico (CVE) rubber collected by producers in Brazil. The advance of farmed rubber has put an end to rubber extraction from the Amazon forests due to a lack of competitiveness. CVE is a new rubber type composed of small clots, clean and odorless, made from latex harvested daily. CVE is easy to manufacture, of good quality, and added value that comes directly from rubber tappers to the industry. According to tests performed, the results show that the tensile-deformation representation curve has a traction resistance limit of $0.54 \mathrm{MPa}$ and that the specific deformation is close to $767 \%$. CVE showed a quality equivalent to that of the Granulado Escuro Brasileiro (GEB) and the Ribbed Smoke Sheet (RSS). It is concluded that CVE can eliminate the prepreparation phase of raw rubber made in GEB plants.
\end{abstract}

Keywords: extractive natural rubber; sustainable development; environment; footwear industry; environmental service 


\section{INTRODUCCIÓN}

La selva amazónica es un bioma único con gran diversidad de animales y vegetales preservado por los pueblos indígenas que lo habitan y fundamental para el planeta por su papel en la regulación del clima (Ellwanger et al., 2020; Stropp et al., 2020; Fearnside, 2021). Particularmente la selva amazónica brasileña es más grande que muchos países del mundo y con más de cinco millones de kilómetros cuadrados, su hidrografía representa el "40\% del territorio brasileño y el $60 \%$ de la disponibilidad de agua del país" (Silva y Bampi, 2019, p. 342). Con una superficie tan extensa, desde el océano Atlántico, en el este, hasta el pie de la cordillera de los Andes, al oeste, tendría que haber algo de gran interés mundial que se produjese en este enorme espacio y que se extendiese por esta inmensa zona verde. Este producto de integración regional es el caucho extraído del Hevea brasiliensis o árbol del caucho.

El caucho natural (NR en acrónimo en inglés) proviene de varias especies de Hevea, siendo un polímero compuesto principalmente por isopreno $\left(\mathrm{C}_{5} \mathrm{H}_{8}\right)$ y otros componentes como proteínas, minerales, carbohidratos y lípidos. Estos componentes proporcionan elasticidad, resiliencia y tenacidad a los compuestos del caucho (Thi Thuong, Trung Nghia y Kawahara, 2018; Sant'Anna et al., 2021). A Charles Goodyear se le atribuye el desarrollo del proceso de vulcanización, que estabiliza el caucho para que no se funda con el calor y se agriete con el frío (Raue et al., 2014, Katzenberg y Tiller, 2016; Junkongab et al., 2020). El NR no vulcanizado posee baja resistencia, es instable en altas y bajas temperaturas e tiene problemas para recuperar su forma después de larga deformación, requiriendo por esto la vulcanización, un proceso físico-químico que convierte el caucho viscoso en redes reticuladas elásticas tridimensionales, resultando em productos menos sensitivos a el calor o frío, con elasticidad, resistencia e estabilidad (Hayeemasae y Masa, 2020). Ese descubrimiento aumentó la demanda por este producto, fomentando la migración poblacional en la selva amazónica. Pero con la domesticación en los cultivos racionales y los polímeros sintéticos, miles de recolectores de caucho han quedado en la selva sin perspectiva de rienda, sin mercado para su producto histórico, el caucho de extracción. Cabe destacar que, por la forma de obtención de este caucho, varios investigadores lo consideran como la mejor forma de protección del bosque donde se introducen. El concepto de "reserva extractiva" como un modelo de área protegida para el uso sostenible, elaborado por los recolectores de caucho, tenía como objetivo garantizar el derecho a la tierra y proteger los bosques de los que extraían su sustento (Wallace et. al, 2018; Brown y Rosendo, 2000).

Incluso con la creación de varias reservas extractivas, solo en agosto de 2018 se contaba con 76 nuevas áreas y 14 millones de hectáreas, siendo éste el modelo de área protegida con conservación y desarrollo para asegurar tierras para los dependientes de los bosques y contener el avance de la deforestación en la región (Gomes et al., 2018), lo que sigue creciendo vertiginosamente es la deforestación, pues solo la creación de reservas no garantiza la supervivencia de las poblaciones. El caucho siempre ha jugado un rol social y económico único en los hogares dedicados a su extracción, porque dada la condición de aislamiento natural, no eran productos perecederos y tenían mercados garantizados (Almeida, 2002; Wallace et. al, 2018).

Por lo tanto, se requiere reconocer y remunerar el servicio medioambiental puro y duro, sin necesidad de deforestar, ni siquiera a través de la "gestión forestal". West y Fearnside (2021) cuestionan la forma en que se pueda mantener la producción de caucho si no existe competitividad para el producto. Para abordar esta cuestión, se ha desarrollado una nueva metodología para la producción de caucho seco extractivo, con cambios mínimos en el proceso de producción y que, en teoría, permite eliminar una etapa clásica de preindustrialización, permitiendo al productor, por primera vez en su trágica historia, ganar un mayor valor por su producto, que, teóricamente, ahora puede venderse directamente a la industria e ir directamente a las mezcladoras para ser vulcanizado (Samonek et al., 2016). Así, para que la selva siga siendo protegida por los pueblos tradicionales que la habitan, es necesario que el producto que permite eso (el caucho) sea valorado, exista un mercado para él (producto) y esto permita al recolector extractivo ejercer su papel de guardabosques, siendo remunerado por el servicio ambiental prestado en función de la cantidad de producto anual entregado a la industria. Con ello se consigue un desarrollo sostenible, en el que el hombre puede recibir el precio justo por su servicio y el medio ambiente permanece sin impactos significativos (Peres y Pastore, 2019).

El caucho Seringô o CVE, es similar al Cernambi Virgem a Granel - CV, llamado también como "Coágulo de Taza". Sin embargo, sólo se diferencia de ésta por el hecho de que el látex debe recogerse a diario y no coagularse en la taza adherida al árbol. En la unidad productiva familiar, este látex se coagula en las mismas tazas utilizadas para su recolección, cuyos coágulos, acomodados en bandas, se secan a la sombra de forma natural, sin necesidad de ser calandrados con las máquinas de presión o de ser secados en hornos de calefacción. Este nuevo proceso genera un coágulo de 20 a $50 \mathrm{~g}$ de caucho, secado a temperatura ambiente, que no necesita de ningún otro procesamiento para ser industrializado. Los recolectores necesitan solamente 3 horas para obtener el caucho Seringô, entre la extracción, la recolección y la producción de los coágulos. Los cuales se quedan remojados en agua clorada, para que al día siguiente se retiren y almacenen en un banco o cinta transportadora para su secado. Después de 10 días a la sombra están listos para ser enviados 
a la fábrica. Actualmente son producidos en el estado de Pará, por aproximadamente 100 familias en la ciudad de Anajás y otras 100 familias en la Reserva Extractiva Tapajós-Arapiuns, en la ciudad de Santarém. El caucho resultante de este nuevo sistema se utiliza en la producción de suelas, plantillas y contrafuertes de sandalias y tenis.

Este trabajo es la primera evaluación en laboratorio científico del CVE/Caucho Seringô, que fue analizado y comparado con el Granulado Escuro Brasileiro - GEB-1 y la Ribbed Smoked Sheet - RSS-3, el primero derivado de una forma de pre-proceso industrial y la segunda de un caucho producido en las plantaciones, ambos destinados para el uso en las industrias de procesamiento de caucho. Las fábricas de GEB-1 sirven para lavar el caucho bruto procedente de las plantaciones de caucho y dejarlo en condiciones para ser procesado en las instalaciones finales. Estas fábricas son caras y costosas, utilizan una enorme cantidad de agua y son altamente contaminantes. Además, consumen mucha energía al utilizar maquinaria pesada y queman madera en los procesos de secado del caucho.

\section{OTROS ANTECEDENTES}

Hay una serie de antecedentes adicionales que es necesario detallar para documentar en mejor forma este trabajo: i) el proceso de obtención del CVE; y ii) los otros cauchos.

\section{El proceso de obtención del CVE}

El caucho Seringô o CVE, es similar al Cernambi Virgen a Granel - CV, llamado también como "Coágulo de Taza". Sin embargo, sólo se diferencia de ésta por el hecho de que el látex debe recogerse a diario y no coagularse naturalmente en la taza adherida al árbol, donde el caucho se sigue degradando por muchos días, corte después de corte. En el sistema tradicional se daña los árboles de caucho hasta que pase la corteza y llegue a los vasos laticíferos, haciendo con que el látex fluya hacia una taza recolectora. Para esto, el sistema se utiliza de un día de corte por dos días de descanso, dando tiempo para que el árbol se rehaga. En el sistema de producción CVE el ciclo es el mismo, pero para mantener-se el látex en estado líquido dentro de la taza hasta su recolección en el mismo día, se utiliza un pequeño chorro de agua de ceniza, un producto alcalino resultante del lavado de cenizas domésticas y que elimina el uso tradicional amoníaco. Esto permite que el caucho se mantenga en estado líquido hasta su recogimiento.

Llegando en la unidad productiva familiar, este látex se coagula en tazas iguales a las utilizadas para su recolección con o uso de un pequeño chorro de ácido acético (vinagre de alcohol). Después de la coagulación de los coágulos, estos se quedan remojados por 24 horas en hipoclorito de sodio (agua sanitaria común) para neutralizar la acidez y desinfectar el coágulo. Después estos son lavados con agua limpia e exprimidos con las propias manos para retirada del exceso de humedad. Los coágulos son acomodados en bandas, se secan a la sombra de forma natural, sin necesidad de ser calandrados con las máquinas de presión o de ser secados en hornos de calefacción. Este nuevo proceso genera un coágulo de 20 a $50 \mathrm{~g}$ de caucho, blanco, parcialmente seco y limpio, que no necesita de ningún otro procesamiento para ser industrializado. Después de no mínimo 10 días a la sombra están listos para ser enviados a la fábrica. Los recolectores necesitan solamente de un total de 3 horas de trabajo para obtener el caucho Seringô, entre la extracción, la recolección y la producción de los coágulos.

Actualmente el CVE/Seringô es producido en el Estado de Pará, por aproximadamente 100 familias en la ciudad de Anajás y otras 100 familias en la Reserva Extractiva Tapajós-Arapiuns, en la ciudad de Santarém en el mismo Estado brasileño. El caucho resultante de este nuevo sistema se utiliza en la producción de suelas, plantillas y contrafuertes de zapatillas y tenis producidos por la 'Cooperativa de Producao dos Ecoextrativistas Da Amazonia - Coopereco', ubicada en Castanhal/Pará, Brasil.

\section{Los otros cauchos}

Las fábricas de GEB-1 (acrónimo del portugués 'Granulado Escuro Brasileiro') sirven para lavar el caucho bruto i sucio y dejarlo en condiciones para ser procesado en las instalaciones finales. Para esto, las plantas fabriles cortan, trituran y lavan el caucho bruto en varias etapas del proceso productivo, para después secarlo em hornos y prensa-lo en fardos. Estas fábricas son caras y costosas por la maquinaria involucrada en el proceso, utilizan una enorme cantidad de agua y son altamente contaminantes al medio, sea por humo o por los contaminantes. Además, consumen mucha energía eléctrica al utilizar maquinaria pesada y muchas queman madera en los procesos de secado del caucho. Para producir esto tipo de caucho hay padrones internacionales finales que necesitan de ser obedecidos para la certificación como un GEB, una adaptación del padrón Standard Malaysian Rubber (SMR).

Para producir las Ribbed Smoked Sheet - RSS-3 es necesario mantener el caucho liquido con el uso de amonia $\left(\mathrm{NH}_{3}\right)$ desde su extracción buscando-se la preservación en el estado líquido. Después, el látex 
mezclado se diluye con agua ( $15 \%$ de caucho seco - hay que medirse la cantidad del caucho en el medio acuoso) y después se coagula con ácido fórmico (hay que se tener la máxima precisión posible). Tras un almacenamiento de 1 a 18 horas, las hojas gelatinosas resultantes, gruesas y blandas, se enrollan mediante cuatro o seis rodillos para eliminar el agua y alcanzar los $5 \mathrm{~mm}$ de grosor, y el último par de rodillos se surca para producir las características marcas acanaladas. A continuación, las láminas se secan entre 4 a 7 días en cobertizos calentados a unos $60^{\circ} \mathrm{C}$ por el humo de la madera quemada para eliminar toda la humedad (Alves, 2004). Pero hay que destacar el hecho de que este caucho es difícil de producir en el ámbito de la pequeña propiedad y por personas sin estudios, dada la complejidad del proceso. En Tailandia se buscó la producción de RSS-3 en las comunidades, pero la producción consumía más leña por tonelada y el $40 \%$ de lo producido también era de baja calidad. Así, más de la mitad de las cooperativas de RSS-3 en Tailandia están sin funcionar (Promtong, 2020).

\section{METODOLOGÍA}

Para este trabajo se utilizaron siete muestras compuestas que se enviaron para ser concentradas en la planta de la Cooperativa de Produção dos Ecoextrativistas da Amazônia (COOPERECO); las cuales fueron recolectadas al azar de la producción, no identificadas por productor, sino sólo por sitio de producción, siendo una mezcla de varios productores y representando la media general. Una vez separadas por ubicación, las muestras compuestas fueron enviadas a los siguientes laboratorios: el Laboratorio de Ingeniería Mecánica de la Universidade Federal do Vale do São Francisco-UNIVASF, en el campus de Juazeiro, estado de Bahía; el Centro de Tecnología de Polímeros SENAI/CETEPO/RS, en São Leopoldo, estado de Rio Grande do Sul y el laboratorio Terra Análises para Agropecuária Ltda., en Goiânia, estado de Goiás, todos en Brasil, donde cada uno siguió la metodología de ensayo recomendada para cada análisis.

Las pruebas realizadas en el Laboratorio de Ingeniería Mecánica de la Universidade Federal do Vale do São Francisco-UNIVASF (Passos, 2019), tuvieron como objetivo la caracterización física y química de un grupo de materiales vegetales impermeabilizados con caucho de la Amazonia. Se realizaron diferentes pruebas, dentro de las cuales el ensayo de tracción, el cual consiste en aplicar una carga de tracción uniaxial sobre un cuerpo de prueba del material hasta su ruptura. También se realizó el cuerpo de prueba, que es una muestra del material con geometría y dimensiones reguladas por normas técnicas como NBR, ASTM, ISO, ABNT y ASME. La tercera prueba fue el ensayo de compresión, que radica en aplicar una carga de compresión uniaxial sobre un cuerpo de prueba, con forma cilíndrica, provocando un aumento en el diámetro de la sección transversal y se realiza en la máquina universal de ensayos. La última prueba fue la de dureza Shore $A$, que es una prueba realizada para evaluar la dureza de la superficie de los materiales blandos, como los elastómeros, según la norma ASTM D 2240, para esta se utilizó el durómetro como equipo de medición.

En el laboratorio del Centro Tecnológico de Polímeros SENAI/CETEPO (CETEPO, 2020), se efectuaron pruebas en la que cada muestra constó de aproximadamente $850 \mathrm{~g}$ de elastómero no vulcanizado de color caramelo, identificada únicamente por el lugar de origen. Las solicitudes fueron identificadas con los números de Orden de Servicio 779/20 a 785/20, todas ellas con fecha del 27 de abril de 2020. Las pruebas fueron: Contenido de suciedad, Plasticidad de Wallace, Contenido de cenizas de caucho natural, Contenido volátil de caucho natural, todas ellas con preparación previa de la muestra, como se muestra en la tabla 1.

La preparación previa consistió en el procesamiento en mezclador abierto (cilindro), utilizando las siguientes condiciones de procesamiento: Temperatura del cilindro: $100 \pm 5 \stackrel{\circ}{\circ}$; apertura entre cilindros: $1.3 \pm 0.15 \mathrm{~mm}$; Relación de revoluciones: 1 - 1.4; Velocidad del cilindro delantero: $14.0 \mathrm{rpm}$; Velocidad del cilindro trasero: 19.6 rpm. La muestra se picó y se pesó, se pasó tres veces en el cilindro (manta inicial). A continuación, se realizaron otras nueve pasadas en el cilindro. Se realizó un nuevo pasaje para la laminación en una sola manta, que se acondicionó en situaciones ambientales de laboratorio: Temperatura: $23 \pm 2{ }^{\circ} \mathrm{C}$; Humedad Relativa: $50 \pm 5 \%$. Se pesó en $70,94,118,142 \mathrm{~h}$, con una variación del $0.1 \%$. Tras la estabilización, la muestra se sometió a pruebas según los métodos/procedimientos de cada prueba, de conformidad con la tabla 1.

Al laboratorio Terra Análises para Agropecuária Ltda. (TAA, 2020) se enviaron siete kilos de CVE y las muestras ingresaron el 3 de agosto de 2020 y finalizadas el 7 de agosto de 2020. No se envió un resumen de las actividades del laboratorio, pero se le pidió que realizaran los análisis de acuerdo con las determinaciones vigentes para el análisis del contenido de nitrógeno en el caucho, es decir, la norma NBR ISO 1656:2010 de la ABNT. A efectos de comparación, dado que el CVE está destinado directamente a la industria, los resultados de los análisis de laboratorio se compararon con las normas exigidas para el GEB-1. Además, los valores del CVE se compararon con los promedios de los resultados de otros cauchos encontrados por Alves (2004) en un estudio sobre la calidad del caucho de varios clones en una plantación de caucho, como se muestra en la tabla 4. 
Tabla 1: Métodos y procedimientos para las pruebas del CVE.

\begin{tabular}{|l|l|}
\hline Contenido de suciedad & $\begin{array}{l}\text { ABNT NBR ISO 249-1:2010. El resultado de la prueba es la media aritmética de dos } \\
\text { determinaciones. }\end{array}$ \\
\hline Plasticidad de Wallace & ABNT NBR ISO 2007:2010. \\
\hline $\begin{array}{l}\text { Contenido de cenizas } \\
\text { de caucho natural }\end{array}$ & $\begin{array}{l}\text { ABNT NBR ISO 247:2010 - Método A. Temperatura de la mufla: 550 } \pm 25 \text { C. El resultado } \\
\text { de la prueba es la media aritmética de dos determinaciones. }\end{array}$ \\
\hline $\begin{array}{l}\text { Contenido volátil de } \\
\text { caucho natural }\end{array}$ & $\begin{array}{l}\text { ABNT NBR ISO 248-1:2012 - Método del horno, Procedimiento A. El resultado de la } \\
\text { prueba es la media aritmética de dos determinaciones. }\end{array}$ \\
\hline
\end{tabular}

Las características del caucho natural tipo GEB-1 están definidas por la ABNT: Suciedad $=0.10$ (ABNT NBR ISO 249); Ceniza = 0.75 (ABNT NBR ISO 247); Volatilidad = 0.80 (ABNT NBR ISO 248); Nitrógeno $=0.60$ (ABNT NBR ISO 1656); Plasticidad inicial (PO Wallace) Mínimo + 0 (ABNT NBR ISO 2007). Los parámetros para este producto son: plasticidad P0 mínimo 30.00; \% de cenizas máximo 0.75; \% de materiales volátiles máximo 0.80 y; \% de contenido de impurezas máximo $0,10 \%$.

\section{RESULTADOS}

Del Laboratorio de Ingeniería Mecánica de la Universidade Federal do Vale do São Francisco-UNIVASF, Passos (2019) revela que la curva representativa de tensión-deformación obtenida para el CVE tenía un límite de resistencia a la tracción de $0.54 \mathrm{MPa}$ y la deformación específica con cerca de $767 \%$. La curva representativa de tensión-deformación obtenida mediante el ensayo de tracción del caucho procesado mostró un límite de resistencia a la tracción de $0.42 \mathrm{MPa}$, una deformación específica del $274 \%$ y un módulo de elasticidad de $0.63 \mathrm{MP}$. Por otro lado, la deformación específica fue del $50 \%$, con un límite de resistencia a la compresión ( $\mathrm{MPa}$ ) de 0,62, con un módulo de elasticidad del $10 \%(\mathrm{MPa})$ en 0.24 . En promedio, la Dureza Shore encontrada fue de 27.4. Los análisis realizados en el Centro de Tecnología de Polímeros SENAI/CETEPO/RS (2020), identificaron cada uno de los lugares de producción observados en la tabla 2.

El laboratorio Terra Análises para Agropecuária Ltda. (TAA, 2020), en su informe mostrado en la tabla 3, no identificó el lugar de origen de cada muestra, aunque eso no es relevante en este estudio, ya que se trata de un análisis general de la media de varios lugares y no de una competencia entre productores, con una media de 0.36 y una mediana de 0.40 en el contenido del nitrógeno de los siete lotes analizados por este laboratorio.

Tabla 2: Resultados de los ensayos de laboratorio del CVE para suciedad, cenizas, volatilidad y plasticidad.

\begin{tabular}{|l|c|c|c|c|}
\hline \multicolumn{1}{|c|}{ Origen } & $\begin{array}{c}\text { Suciedad } \\
(\%)\end{array}$ & $\begin{array}{c}\text { Cenizas } \\
\text { (\% promedio) }\end{array}$ & $\begin{array}{c}\text { Volatilidad } \\
\text { (\% promedio) }\end{array}$ & Plasticidad (Mediana) \\
\hline Alto Rio Mocoões - Anajás/PA & 0.03 & 0.20 & 0.22 & 52.00 \\
\hline Alto Rio Anajás - Anajás/PA & 0.02 & 0.20 & 1.30 & 52.50 \\
\hline Ig. Purus Anajás/PA & 0.04 & 0.20 & 1.31 & 52.80 \\
\hline Ig. Marinheiro - Anajás/PA & 0.02 & 0.22 & 1.22 & 51.80 \\
\hline V. Franca - Resex Tapajós Arapiuns - Santarém/PA & 0.02 & 0.22 & 1.10 & 51.30 \\
\hline V. Boim - Resex Tapajós Arapiuns - Santarém/PA & 0.04 & 0.20 & 1.42 & 55.00 \\
\hline V. Arajó - Inhangapi /PA & 0.04 & 0.21 & 1.22 & 53.60 \\
\hline Máximo & 0.04 & 0.22 & 1.42 & 55.00 \\
\hline Mínimo & 0.02 & 0.20 & 0.22 & 51.30 \\
\hline Media general & 0.03 & 0.21 & 1.11 & 52.71 \\
\hline Mediana general & 0.03 & 0.20 & 1.22 & 52.50 \\
\hline
\end{tabular}

Tabla 3: Análisis del contenido de Nitrógeno $(\mathrm{N})$ identificados en las muestras de caucho bruto tipo CVE.

\begin{tabular}{|c|c|c|}
\hline Orden & Código & Resultado (N\%) \\
\hline 1 & SAL 82049 & 0.60 \\
\hline 2 & SAL 82050 & 0.40 \\
\hline 3 & SAL 82051 & 0.30 \\
\hline 4 & SAL 82052 & 0.40 \\
\hline 5 & SAL 82053 & 0.20 \\
\hline 6 & SAL 82054 & 0.40 \\
\hline 7 & SAL 82055 & 0.20 \\
\hline Media & & 0.36 \\
\hline Mediana & & 0.40 \\
\hline
\end{tabular}




\section{DISCUSIÓN}

En la comparación directa de los promedios, se verifica que el CVE fue superior en todas las cuestiones analizadas, excepto en el contenido de volátiles ya sea en comparación con los estándares definidos por la ABNT o en comparación con el trabajo de Alves (2004). Por lo que se procede a analizar los resultados individualmente entre el CVE, el GEB-1 y la RSS-3 en comparación con el trabajo desarrollado por el mismo autor.

\section{Suciedad}

A partir de la comparación básica inicial con el GEB-1, el CVE muestra niveles muy superiores en términos de limpieza del producto. Mientras que el GEB-1 exige una superestructura para su limpieza, incluso con un elevado consumo de agua y es considerablemente contaminante, todo ello para alcanzar niveles entre el 0.01 y el $0.10 \%$ de suciedad, con una media del $0.05 \%$, el CVE, sin ningún esfuerzo extra del recolector aparte de un mínimo cuidado en el proceso de obtención del coágulo y un posterior lavado, consiguió obtener valores medianos muy inferiores, de $0.03 \%$ en promedio. Cuando se comparan los valores de suciedad con los de la RSS-3, los promedios del CVE son mejores, lo que demuestra que se trata de un caucho más limpio. Cabe señalar que la metodología para obtener la RSS-3 siempre ha sido un dilema para las comunidades de extractores del caucho, dada la necesidad de controlar estrictamente las etapas del proceso.

En cuanto a la suciedad, los datos revelan que ha habido una excelente asimilación de los conceptos de higiene y cuidado en la elaboración del CVE, ya que prácticamente se ha eliminado la contaminación del producto por suciedad. Esto demuestra que es posible obtener un caucho limpio sin necesidad de costosos procesos de limpieza (GEB-1) o de procesos de producción con un nivel de tecnicidad superior a la capacidad del recolector promedio amazónico. Aquí se produce una inversión del proceso histórico, ya que la práctica habitual de los trabajadores del caucho en el sistema tradicional de explotación esclavista, donde el patrón desempeñaba el papel de esclavizador, una de las opciones era añadir materiales extraños al caucho en diversos objetos. Esto se hacía con harinas, tierra y otros objetos más grandes escondidos dentro de las piezas de goma ahumada (Benchimol, 1992). Ahora, en el nuevo sistema, el recolector del caucho es el jefe de sí mismo y la calidad es parte del producto que se ofrece, situación que él entiende dentro de un nuevo sistema de calificación construido junto con el productor. Esto llevó al empoderamiento del recolector del caucho que, al ser reconocido y valorado, se aplicó de manera más concentrada en la producción del caucho, logrando un producto de altísima calidad y que no deja nada que desear a los mejores productos obtenidos con técnicas refinadas y mayores inversiones.

\section{Contenido de Cenizas}

El bajo contenido de minerales en el caucho CVE es un indicio más de higiene, ya que el caucho en sí tiene pocos minerales, al ser una cadena orgánica. El alto contenido en minerales está relacionado con la contaminación durante el proceso de obtención del coágulo, ya sea en contacto con el suelo o incluso con la presencia de suciedad como hojas, insectos o incluso la adición de arcillas para aumentar el peso de cada coágulo. La determinación de las cenizas revela los componentes inorgánicos no descompuestos a aproximadamente $600{ }^{\circ} \mathrm{C}$, en donde valores altos reducen las propiedades dinámicas del material vulcanizado, influyendo negativamente en las propiedades de envejecimiento (Sant'Anna et al., 2021).

Si el contenido de cenizas se encuentra por encima de los valores adoptados como estándar puede influir negativamente en las propiedades dinámicas del vulcanizado, potenciando las propiedades negativas de envejecimiento (Antonia, 2003). Pero los bajísimos valores obtenidos por el CVE revelan ser un caucho con mínimos valores minerales, demostrando un potencial adecuado para enfrentar el desgaste por envejecimiento, manteniendo sus propiedades por tiempo considerable y de forma superior al GEB-1 común, siendo incluso mejor que la RSS-3 analizada por Alves (2004) y utilizado para las comparaciones. Como se mencionó en el ítem que analizó los niveles de suciedad, no existen motivos adicionales para que el cauchero introduzca productos ajenos al caucho, sino que sea más cuidadoso con su producto, manteniendo la higiene durante el proceso de obtención del coágulo y los procedimientos posteriores, como el lavado adecuado y correcto. Ahora, empoderado de su condición de "guardabosque independiente", entiende su participación en el proceso y ha sido remunerado en consecuencia por estos servicios, dejando de ser un esclavo explotado por un sistema abrasador.

\section{Volátiles-Humedad}

De acuerdo con Wisniewski (1983), el porcentaje de volátiles en el caucho es correlacionado principalmente con el contenido de humedad o la eficiencia de secado, y también puede ser una contaminación por materiales higroscópicos, dado que los demás elementos involucrados son de bajo peso molecular y no analizados en esta etapa del proceso. 
De este modo, hay que destacar que una de las muestras era de excelente calidad, con apenas un $0.22 \%$ de volatilidad, lo que indica un correcto proceso de producción y un adecuado secado. En los otros, se comprueba que es necesaria una intervención para un mayor control del proceso de secado al aire libre o más tiempo de secado, especialmente en los periodos lluviosos de la Amazonia. Y aquí tenemos la respuesta: mientras que el GEB-1 y la RSS-3 se someten a procesos artificiales (invernaderos) para su secado, el CVE se seca a la sombra y al aire libre en una región donde normalmente los índices de humedad son superiores al $96 \%$.

Los resultados están dentro de lo esperado, pero revelan que un poco más de cuidado puede dar lugar a un producto dentro de las características requeridas para un caucho de alta calidad, ya que la mediana estaba un $50 \%$ por encima de la norma internacional en comparación con el GEB-1. De otra manera, la fábrica no tuvo problemas con la humedad presente, dado que los coágulos son nuevamente dejados a secar por un periodo complementar de dos o tres días en espacio cubierto, no siendo detectados problemas en el procesamiento industrial e en la confección del calzado dado los procesos involucrados, como la masticación, calandrado y prensado. Pero si un productor puede alcanzar valores por debajo de la norma máxima con pequeños ajustes en el proceso, además de que, si son unidades relativamente nuevas, las correcciones técnicas se harán a partir de la detección de mínimos errores de procedimiento, que puede solucionarse con un aumento del alero del lugar donde se secan los coágulos y uno o dos días más en el proceso de secado en caso de fuertes lluvias durante el mismo.

Por otro parte, como dijo, la cooperativa que actualmente procesa el CVE complementa el secado al aire libre antes de utilizar el producto en las mezcladoras. Es necesario considerar que la metodología utilizada también elimina los restos de humedad al mezclar los cauchos y los demás componentes para la vulcanización, por lo que estos datos no afectan a la industria compradora. Pero es necesario considerar que lo ideal es obtener valores más bajos y que todos los productores traten de secar el producto de forma más adecuada y por más tiempo para obtener la certificación primaria.

\section{Plasticidad/PO}

La plasticidad de Wallace (P0) está relacionada con la longitud de la cadena de poliisopreno o caucho natural, y se considera como una evaluación de la degradación del caucho natural, ofreciendo una visión de la microestructura del material en su viscosidad. Su medición proporciona una estimación de la longitud de la cadena del polímero y del estado de degradación de la materia prima (Samantarai et al., 2019; Sant'Anna et al., 2021). En cuanto a la plasticidad/PO mediana, los valores encontrados en el CVE muestran un comportamiento estándar y común a todas las muestras analizadas, variando muy cerca de la mediana y sin grandes oscilaciones. Por otro lado, al tratarse de un caucho natural y sin ninguna preparación previa, el producto tiene una plasticidad ligeramente inferior, pero todavía por debajo de las normas internacionales si se compara con el GEB-1. La plasticidad final del GEB-1 es una función del estándar requerido por el comprador y un resultado de la mezcla de varios cauchos. Tanto es así que las pruebas realizadas por Alves (2004) revelaron altos valores de plasticidad. EI CVE que, a pesar de ser más bajo en este aspecto, está muy cerca del GEB-1 y lejos de la norma mínima (30.00), lo que indica que son gomas similares en este aspecto.

Es importante destacar que la RSS-3 es más agresiva para el medio ambiente y para el hombre, porque es el resultado del ahumado de las cuchillas, que implica otros procesos nocivos, por ejemplo, en la manipulación del amoníaco como anticoagulante y procesos de preparación agotadores, que se observan en los diversos pasos del coágulo por las calandras. Es muy probable que la forma de producción del CVE no afecte a las mejores características de un caucho natural de calidad, pero para conseguirlo no ha sido necesario realizar cambios drásticos en el modo de producción, sino sólo la comprensión del proceso y un poco de cuidado en la producción, situaciones al alcance de cualquiera y que no requieren altos conocimientos ni inversiones que estén fuera del alcance del recolector de caucho promedio.

\section{Nitrógeno/Proteínas}

En relación al análisis de los niveles de nitrógeno $(\mathrm{N})$, se reveló que de las siete muestras enviadas al laboratorio sólo una presentaba resultados que se aproximaba a los niveles máximos recomendados por la norma NBR ISO 1656:2010 de la ABNT, que determina un máximo de $0.60 \%$ de proteínas presentes en los cauchos tipo GEB-1. Los datos muestran que el CVE presentó una media de $0.36 \%$ y una mediana de $0.40 \%$, con un extremo mínimo de $0.20 \%$ y el máximo ya mencionado de $0.60 \%$. Los contenidos máximos de nitrógeno encontrados en las muestras analizadas revelan un caucho de calidad, sin efectos deletéreos posteriores en relación con las propiedades de resistencia durante la vulcanización. Por otro lado, los valores mínimos también están dentro de los límites aceptados, es decir, entre el $0.2 \%$ y el $0.6 \%$ (Antonia et al., 2003). Los cauchos CVE analizados son de buena calidad porque presentan contenidos de nitrógeno dentro del margen aceptable, con media y mediana dentro del espectro mencionado anteriormente y como se aprecia en el análisis de los datos de la tabla 4, del Granulado Escuro Brasileiro (GEB-1), la Ribbed Smoked Sheet (RSS-3) y el Cernambi Virgem Ecológico (CVE). 
Tabla 4: Datos analizados de los tres cauchos: GEB-1, RSS-3 y CVE.

\begin{tabular}{|l|l|c|c|c|c|c|}
\hline Identificación & Dato & $\begin{array}{c}\text { Suciedad } \\
\text { (\%) }\end{array}$ & $\begin{array}{c}\text { Cenizas } \\
\text { (\% promedio) }\end{array}$ & $\begin{array}{c}\text { Volatilidad } \\
\text { (\% promedio) }\end{array}$ & $\begin{array}{c}\text { Nitrógeno } \\
\text { (Máximo 0.6\%) }\end{array}$ & $\begin{array}{c}\text { Plasticidad } \\
\text { (Mediana) }\end{array}$ \\
\hline \multirow{5}{*}{ GEB-1 } & ABNT & 0.10 & 0.75 & 0.80 & 0.60 & 30.00 \\
\cline { 2 - 7 } & Máximo & 0.70 & 0.53 & 0.67 & 0.47 & - \\
\cline { 2 - 7 } & Mínimo & 0.01 & 0.18 & 0.33 & 0.20 & - \\
\cline { 2 - 7 } & Media & 0.10 & 0.30 & 0.30 & 0.30 & - \\
\cline { 2 - 7 } & Mediana & 0.05 & 0.25 & 0.44 & 0.30 & 60.33 \\
\hline \multirow{5}{*}{ RSS-3 } & Máximo & 0.10 & 0.58 & 1.15 & 0.57 & - \\
\cline { 2 - 7 } & Mínimo & 0.01 & 0.24 & 0.57 & 0.25 & - \\
\cline { 2 - 7 } & Media & 0.06 & 0.37 & 0.59 & 0.41 & - \\
\cline { 2 - 7 } & Mediana & 0.06 & 0.39 & 0.69 & 0.40 & 88.18 \\
\hline \multirow{5}{*}{ CVE } & Máximo & 0.04 & 0.22 & 1.42 & 0.60 & 55.00 \\
\cline { 2 - 7 } & Mínimo & 0.02 & 0.20 & 0.22 & 0.20 & 51.30 \\
\cline { 2 - 7 } & Media & 0.03 & 0.21 & 1.11 & 0.36 & 52.71 \\
\cline { 2 - 7 } & Mediana & 0.03 & 0.20 & 1.22 & 0.40 & 52.50 \\
\hline
\end{tabular}

\section{CONCLUSIONES}

La nueva forma de producción de caucho, el CVE o "caucho Seringô", no presenta dificultades para su implementación por parte de los caucheros amazónicos, ya que se trata de una Tecnología Social que fue desarrollada, sistematizada y transmitida por Poloprobio, una ONG amazónica y certificada por la Fundación Banco do Brasil como Tecnología Social. El caucho extraído por este método tiene un gran potencial para sustituir tanto al GEB-1 como al RSS-3 para servir a nichos de mercado, especialmente a la industria del calzado. Actualmente las grandes marcas están haciendo la transición en sus líneas de producción para la sostenibilidad, dejando de utilizar derivados del petróleo y volviendo al caucho natural, por ser una materia prima más sostenible. Asimismo, los resultados de los análisis de laboratorio demuestran que se trata de un producto limpio, con una flexibilidad intermedia, cuya composición no implica sacrificios ni daños en los equipos necesarios para su manipulación. Por otro lado, por ser un caucho que se puede comercializar directamente con la industria, eliminando intermediarios y comisionistas, permitió una mayor remuneración para los productores, por kilo de producto. La fábrica de Coopereco no tuvo problemas y ya está comercializando suelas y calzado deportivo y se encuentra en la fase final de pruebas. El nuevo sistema de producción de caucho crudo extraído tiene la calidad suficiente para ser una forma de integrar el crecimiento financiero de las comunidades productoras de caucho con la protección del medio ambiente en el que viven. En promedio, cada productor tiene una superficie de $1,5 \mathrm{~km}^{2}$ y una producción media de $330 \mathrm{~kg}$ de caucho al año con humedad, lo que permite inferir que por $2,2 \mathrm{~kg}$ de caucho seco vendido, se está protegiendo una superficie de $0,1 \mathrm{~m} / \mathrm{km}^{2}\left(10.000 \mathrm{~m}^{2}\right)$ de bosque nativo. Esto es el desarrollo sostenible.

\section{REFERENCIAS}

Almeida, M.B.d., The Politics of Amazonian Conservation: The Struggles of Rubber Tappers, https://doi.org/10.1525/jlca.2002.7.1.170, Journal of Latin American Anthropology, 7(1), 170-219 (2002)

Alves, M., Estudo da borracha natural para utilização em períodos de entressafra num mesmo composto, Tesis de Maestría en Ingenieria Quimica, , Universidade Estadual de Campinas, Campinas, São Paulo, Brasil, http://repositorio.unicamp.br/bitstream/REPOSIP/266144/1/Alves_MarciaReginadaCosta_M.pdf/, (2004)

Antonia, A., Estudo do desempenho mecânico e térmico de compostos de borracha natural (NR) de diferentes clones, Universidade de São Paulo, São Carlos, SP, Brasil, https://doi.org/10.11606/D.88.2015.tde-24062015-194239 (2003)

Benchimol, S., Romanceiro da Batalha da Borracha, Manaus: Imprensa Oficial Governo do Estado do Amazonas (1992)

Brown, K., y Rosendo, S., The Institutional Architecture of Extractive Reserves in Rondônia, Brazil, https://doi.org/10.1111/j.1475-4959.2000.tb00005.x, Geographical Journal, 166(1), 35-48 (2000)

CETEPO, Centro Tecnológico de Polímeros, Relatório de ensaios, SENAI, São Leopoldo, Rio Grande do Sul, Brasil (2020)

Ellwanger J. H., y otros 13 autores, Beyond diversity loss and climate change: Impacts of Amazon deforestation on infectious diseases and public health, https://doi.org/10.1590/0001-3765202020191375, Anais da Academia Brasileira de Ciências, 92(1:e20191375), 1-33 (2020)

Fearnside, P.M., The intrinsic value of Amazon biodiversity, https://doi.org/10.1007/s10531-021-02133-7, Biodiversity and Conservation, 30(4), 1199-1202 (2021)

Gomes, C., y otros 3 autores, Extractive Reserves in the Brazilian Amazon thirty years after Chico Mendes: social movement achievements, territorial expansion and continuing struggles, https://doi.org/10.5380/dma.v48i0.58830, DMA: Desenvolvimento e Meio Ambiente, 48, Edição especial: 30 Anos do Legado de Chico Mendes, 74-98 (2018) 
Hayeemasae, N., \& Masa, A., (2020). Relationship between stress relaxation behavior and thermal stability of natural rubber vulcanizates. Polímeros: Ciência e Tecnologia, 30(2), e2020016. https://doi.org/10.1590/0104-1428.03120.

Junkongab, P., y otros 5 autores, Effect of fatty acids on the accelerated sulfur vulcanization of rubber by active zinc/carboxylate complexes, https://doi.org/10.1039/C9RA10358A, RSC Advances, 10(8), 4772-4785 (2020)

Katzenberg, F., y Tiller, J.C., Shape memory natural rubber, https://doi.org/10.1002/polb.24040, Journal of Polymer Science, Part B: Polymer Physics, 54(14), 1381-1388 (2016)

Passos, L., Caraterização física e química de um grupo de materiais encauchados de vegetais da Amazônia: Relatório técnico final, Universidade Federal do Vale do São Francisco, Campus Juazeiro-BA (2019)

Peres, J. B. R., Jr., y Pastore, F., Jr., Amazon rubber, a potential yet to be rediscovered, https://doi.org/10.1007/s10924019-01381-7, Journal of Polymers and the Environment, 27(3), 652-658 (2019)

Promtong, M., Two-stage forced convection furnace for ribbed Rubber Smoked Sheet (RSS) production: performance evaluation, https://doi.org/10.21660/2020.68.9186, International Journal of GEOMATE, 18(68), 120-128 (2020)

Raue, M., Wambach, M., y otros 9 autores, Investigation of Historical Hard Rubber Ornaments of Charles Goodyear, https://doi.org/10.1002/macp.201300629, Macromolecular Chemistry and Physics, 215(3), 245-254 (2014)

Samantarai, S., Nag, A., y otros 5 autores, Chemical modification of nitrile rubber in the latex stage by functionalizing phosphorylated cardanol prepolymer: A bio-based plasticizer and a renewable resource, https://doi.org/10.1177/0095244318768644, Journal of Elastomers \& Plastics, 51(2), 99-129 (2019)

Samonek, F., y otros 3 autores, Encauchados 20 anos - do Acre para o mundo. Coleção Encauchados de vegetais da Amazônia - Livro 1, 172, Gráfica Supercores, Belém, Brasil (2016)

Sant'Anna, I.C., y otros 5 autores, Genetic diversity associated with natural rubber quality in elite genotypes of the rubber tree, https://doi.org/10.1038/s41598-020-80110-w, Scientific Reports, 11(1081), 1-10 (2021)

Silva, C. A. F., y Bampi, A. C., Regional Dynamics of Brazilian Amazon: between Modernization and Land Conflicts, https://doi.org/10.15446/rcdg.v28n2.72872, Cuadernos de Geografía: Revista Colombiana de Geografía, 28(2), 340-356 (2019)

Stropp, J., Umbelino, B., y otros 4 autores, The ghosts of forests past and future: deforestation and botanical sampling in the Brazilian Amazon, https://doi.org/10.1111/ecog.05026, Ecography, 43(7), 979-989 (2020)

TAA, Terra Análises para Agropecuária Ltda., Resultado de Análise laboratorial, Goiânia, Goiás, Brasil (2020)

Thi Thuong, N., Trung Nghia, P., y Kawahara, S., Factors influencing green strength of commercial natural rubber, https://doi.org/10.1515/gps-2018-0019, Green Processing and Synthesis, 7(5), 399-403 (2018)

Wallace, R.H., Gomes, V.C.A., y Cooper, N.A., The Chico Mendes Extractive Reserve: trajectories of agroextractive development in Amazonia, https://doi.org/10.5380/dma.v48i0.58836, DMA: Desenvolvimento e Meio Ambiente, 48, Edição especial: 30 Anos do Legado de Chico Mendes, 184-213 (2018)

West, T.A.P., y Fearnside, P.M., Brazil's conservation reform and the reduction of deforestation in Amazonia, https://doi.org/10.1016/j.landusepol.2020.105072, Land Use Policy, 100, 1-12 (2021)

Wisniewski, R., Látex e borracha (Informe Técnico, 4), Faculdade de Ciências Agrárias do Pará, Belém, Pará, Brasil (1983) 
